\title{
Mitochondrial dysfunction, oxidative stress, and major depressive disorder
}

This article was published in the following Dove Press journal:

Neuropsychiatric Disease and Treatment

25 April 2013

Number of times this article has been viewed

\section{Edward H Tobe}

University of Medicine and Dentistry of New Jersey/School of Osteopathic Medicine, University of Medicine and Dentistry of New Jersey/Robert Wood Johnson Medical School, and Cooper Medical School of Rowan University, Camden, NJ, USA
Correspondence: Edward H Tobe 100I Lincoln Drive West Suite B, Marlton, NJ 08053-1534, USA

Tel +l 8569834940

Email edward.tobe@comcast.net
Abstract: There is controversy about depression being a physical illness, in part because a reproducible, sensitive, and specific biologic marker is not available. However, there is evidence that mitochondrial dysfunction and oxidative stress may be associated with abnormal brain function and mood disorders, such as depression. This paper reviews selected human and animal studies providing evidence that intracellular mitochondrial metabolic dysfunction in specific brain regions is associated with major depressive disorder. This supports the hypothesis that chronic mitochondrial dysfunction in specific tissues may be associated with depression. Evaluation of mitochondrial dysfunction in specific tissues may broaden the perspective of depression beyond theories about neurotransmitters or receptor sites, and may explain the persistent signs and symptoms of depression.

Keywords: mitochondrial dysfunction, oxidative stress, major depressive disorder, depression

\section{Introduction}

Most human pharmacologic investigations of depression start with criteria for major depressive disorder (MDD) from the Diagnostic and Statistical Manual of Mental Disorders. ${ }^{1}$ The overlap of signs and symptoms in primary or secondary mental disorders generates multiple diagnoses called comorbidities. A meta-analysis showed that it was difficult to differentiate between placebo and medication response unless the Hamilton Depression Rating Scale score was $>23,{ }^{2,3}$ but patients who were more severely ill benefited from medication more than placebo. Serendipitous research findings may have value, such as the antidepressant response in patients who received iproniazid to treat Mycobacterium tuberculosis, but such findings may be limited in contributing to the design of new treatments for depression. ${ }^{4}$

The cause of depression is complicated by language. The term "depression" is descriptive and is not based on biologic findings. It has parallels in language history that convey descriptive states, such as deprimere (Latin, meaning "to press down"), lugubrious from lugere (Latin, meaning "to mourn"), and melancholy from melagkholiā (Greek, meaning "sadness", from melas and khole ["black bile"]).

Previous studies have demonstrated abnormal mitochondrial function and oxidative stress in various diseases. EHT hypothesized that intracellular mitochondrial metabolic dysfunction in specific brain regions represents either a cause or effect of MDD. The purpose of the present study was to review the literature for evidence to evaluate this hypothesis. 


\section{Mitochondrial function}

The brain adapts to environmental or internal challenges with alterations in synaptic efficacy, promotion of neurogenesis, suppression of pathologic apoptosis, and neuronal molding. All human cellular functions require energy that is generated in numerous mitochondria per cell. If mitochondrial DNA (mtDNA) becomes compromised, various cascades of dysfunctional metabolism may impair cellular connectivity and communication.

Mitochondria oxidize glucose through an oxidative phosphorylation system composed of five of the 13 subunits of mtDNA. These complexes exist in the inner mitochondrial membrane. The energy released during electron transport helps shift protons across the inner mitochondrial membrane, decreasing the concentration of protons on the inside of the membrane and creating gradients of acid-base and potential energy. The energy generated by protons spontaneously diffusing across the inner membrane is used to convert adenosine diphosphate to adenosine triphosphate. Mitochondria are the site of most cellular production of adenosine triphosphate.

Reactive oxygen species are free radicals (molecules with one unpaired electron) derived from molecular oxygen. In its lowest energy state, molecular oxygen $\left(\mathrm{O}_{2}\right)$ has two unpaired electrons with similar spin orientation in its outer electron orbit. If one of the unpaired electrons is excited, the electron orbit changes and the oxygen molecule becomes more reactive. Mitochondrial oxidative phosphorylation involves respiratory complexes that donate electrons to oxygen, producing superoxide anions and peroxides.

Excessive reactive oxygen species can damage intracellular proteins and lipids, oxidize mtDNA and nuclear DNA, and open the mitochondrial permeability transition pore to cause mitochondrial swelling and release of apoptotic effectors. If the mitochondrial outer membrane ruptures, the apoptotic factor cytochrome $\mathrm{c}$ is released. Reactive oxygen species may function as an intracellular signal transduction regulator, ${ }^{6,7}$ and this exemplifies the subtlety of the balance of intracellular reactive oxygen species.

Apoptosis is induced by either extrinsic stimulation of specific death receptors on the cell membrane or intrinsic mechanisms derived from mitochondrial stress. Apoptosis is tightly regulated, and increased or decreased apoptosis may cause cancer or developmental, autoimmune, or neurodegenerative diseases. ${ }^{8}$

The location of mitochondria is determined by energy need. The endoplasmic reticulum forms a network of tubules and cisternae throughout the cytoplasm, with an inhomogeneous distribution of calcium uptake and release sites. Cytoplasmic signals may converge in the endoplasmic reticulum to form spatiotemporally controlled patterns of calcium release. ${ }^{7}$ In brain cells, the endoplasmic reticulum has two release channels for calcium ions, ie, inositol 1,4,5-triphosphate receptors and ryanodine receptor type 3 . Mitochondria linked to the inositol 1,4,5-triphosphate receptor and ryanodine receptor channels control local calcium release and cytoplasmic calcium regulation. ${ }^{9}$

Mitochondria are involved in numerous metabolic activities, including the urea cycle, lipid metabolism, porphyrin synthesis, and homeostasis of steroid hormones and cellular calcium ions. Calcium ions stimulate oxidative phosphorylation, upregulate the creation of adenosine triphosphate, and affect the metabolism of other molecules. Calcium ions enter the mitochondria by a well regulated outer and inner mitochondrial membrane process. The efflux of calcium ions is dependent on sodium exchange. Glutamate stimulates plasma membrane influx of calcium ions. Glutamate synapses are tripartite, involving the presynaptic neuron, postsynaptic neuron, and glial cell.

There are obstacles to demonstrating mitochondrial dysfunction that may predict a depressed, lugubrious, melancholic mental state. Human research is compromised by genetic variation, environmental and pharmacologic exposures, and comorbid diagnosed or undiagnosed illnesses in controls and patient groups. Furthermore, extrapolation of data from animal research must be done with caution.

\section{Clinical and imaging studies}

Infection is an illness that permits an observable cause-effect relationship. A study of survival or death from sepsis highlighted the importance of mtDNA haploids. ${ }^{10}$ Mitochondrial DNA is composed of maternally inherited circular haploid groups, named from $A$ to $Z$ in the sequence of their discovery. If mtDNA becomes compromised, cascades of dysfunctional metabolism may impair cellular connectivity and communication. There is a "physiologic reserve" that is affected by mtDNA. ${ }^{10}$ In 150 severely ill patients with sepsis who were admitted to an intensive care unit, there was a 2.12 -fold ( $95 \%$ confidence interval 1.02-4.43) increased likelihood of survival at 180 days in patients with haploid group $\mathrm{H}$ than patients without this haploid group..$^{10}$ This suggested that genetic haploids may provide a "physiologic reserve" that is protective against physiologic stress. This finding is similar to the observation that the presence of certain genetic material permits a physiologic reserve in transgenic rats. Genetic haploids may determine the degree of vulnerability to certain diseases, such as depression. 
Functional imaging to study brain metabolism is based on mitochondrial respiration that requires glucose, but neurons do not store glucose. Glucose is transported across the plasma membrane of a cell by a glucose transporter protein (GLUT) that has several isoforms in different tissues. The isoform GLUT1 transports glucose and other hexoses across the blood-brain barrier and astrocyte plasma membrane, and the isoform GLUT3 transports glucose into the neuron. ${ }^{11}$ Positron emission tomography uses ${ }^{18} \mathrm{~F}$-fluorodeoxyglucose, an analog of glucose that emits positrons when glucose is metabolized within the cell. The brain regulates its blood supply according to metabolic need. Single-photon emission computed tomography images brain metabolism using selected radiopharmaceuticals that emit gamma rays. Magnetic resonance imaging (MRI) provides images of the anatomy of the brain. Imaging studies from positron emission tomography, single-photon emission computed tomography, and MRI scans were evaluated to determine anatomic and metabolic correlates with MDD. ${ }^{12}$ In patients with a mood disorder, data were reviewed about regional changes in brain metabolism, volume, and structure. Regions demonstrating brain pathology included the medial prefrontal cortex, medial and caudolateral orbital cortex, amygdala, hippocampus, ventromedial parts of basal ganglia, and anterior cingulate cortex. ${ }^{12}$

In another study, the amygdala was the only structure in which regional blood flow and glucose metabolism correlated with the severity of depression. ${ }^{13}$ There was increased metabolism in the amygdala during wakefulness and sleep. In asymptomatic periods between depressive episodes, patients with familial depression who were not receiving antidepressant treatment showed abnormally elevated blood flow and metabolism; in contrast, patients with familial depression who were receiving antidepressant treatment had normal amygdala metabolism. ${ }^{13}$

A study with MRI scans evaluated orbital frontal cortex volume in 15 patients in remission from MDD and 20 control subjects. ${ }^{14}$ Patients in remission from MDD had a $32 \%$ smaller volume of the medial orbitofrontal cortex than the control subjects. ${ }^{14}$ The ongoing volume loss may have occurred because of impaired anatomic recovery from MDD, and this suggested that MDD may cause chronic brain tissue changes.

Cerebellar dysregulation may be a primary or secondary manifestation of mood disorder. Abnormal affect and cognitive impairment may occur in children and adults with cerebellar cognitive affective syndrome. ${ }^{15}$ The vermis and fastigial nucleus function as a "limbic cerebellum". This concept is supported by neuropsychologic testing of adults with lesions confined to the cerebellum, children with posterior fossa syndrome, patients with developmental anomalies of the cerebellum, and patients with spinocerebellar ataxia. ${ }^{15}$

Another MRI study evaluated 16 patients hospitalized for the first time with a manic episode, 14 patients with prior hospitalizations for manic episodes, and 15 control subjects. ${ }^{16}$ The right and left vermal areas 1-3 were measured. Cerebellar vermal area 3 (vermal lobules VIII-X) was smaller in patients with bipolar disorder who experienced multiple episodes of depression. ${ }^{16}$ Therefore, cerebellar vermal atrophy may be a late neurodegenerative event in patients with multiple affective episodes.

A post mortem study supports the importance of coexistent damage to the cerebellum and temporal lobe. In a middle-aged man with depression, dementia, and volatile behavior, histology demonstrated partial necrosis of the temporal lobe, disorganized collection of large neurons and granular cells in the vermis nodulus, and excessive numbers of Bergmann astrocytes, probably the result of a closed head injury. ${ }^{17}$

Extensive clinical diagnostic screening of women (aged 23-86 years) with a history of recurrent MDD without medical comorbidity and normal control subjects showed that there was no difference between the two subject groups in cortisol levels obtained the day before an oral dexamethasone $(1 \mathrm{mg})$ suppression test. ${ }^{18}$ Evaluations confirmed that neither control subjects nor patients in this study had current depression. There was no relationship between age and volume loss of the hippocampus, but there was a direct relationship between total lifetime depression duration, bilateral hippocampus volume loss, and smaller volume of the amygdala core nuclei. Women with recurrent MDD in remission had verbal memory loss. ${ }^{18}$

\section{Human histologic studies}

In two post mortem histologic studies of brain tissue from people who had MDD, the diagnosis of MDD was confirmed using information from different sources, but the diagnosis of MDD may be unreliable because of unknown personal and medical factors. ${ }^{19,20}$

A histologic study in humans showed morphometric evidence of neuronal and glial cell changes in patients with MDD. ${ }^{19}$ There had been functional abnormalities found on imaging the left dorsolateral prefrontal cortex and left orbitofrontal cortex. Patients with a retrospective diagnosis of MDD without psychosis were compared with normal control subjects. In patients with MDD, there was diminished neuronal size and cortical thickness (most prominently in the rostral orbitofrontal region), markedly diminished glial 
density, and moderately diminished neuronal size, without significant loss of cortical thickness in the left caudal orbitofrontal region and dorsolateral prefrontal cortical region. ${ }^{19}$

Post mortem brain tissue was evaluated from area $24 \mathrm{~b}$ of the supracallosal anterior cingulate cortex in subjects with MDD, bipolar disorder, or schizophrenia, and in normal control subjects. Subjects with MDD had decreased glial cell density $(22 \%, P=0.004)$ and neuronal size $(23 \%, P=0.01)$ in layer 6 than control subjects. ${ }^{20}$

\section{Animal studies}

Monoamine oxidase is an enzyme attached to the outer membrane of the mitochondria. Diverse monoamine oxidase activity has been observed in purified beef brain mitochondria, and enzyme properties are influenced by substrate and $\mathrm{pH} .^{21}$ The mitochondrial membrane may restrict enzymatic activity for different substrates, and this may be affected by $\mathrm{pH}$ and ion concentrations. ${ }^{21}$

In a study of $\mathrm{N}$-methyl-D-aspartate (NMDA) receptor activation in neonatal rat cardiomyocytes, overstimulation of NMDA receptors by glutamate caused excessive calcium ion influx, which compromised mitochondrial membrane polarity; glutamate overstimulation also caused an increase in reactive oxygen species and a cascade of apoptotic factors. ${ }^{22}$ Tianeptine modulated the glutamatergic effects at NMDA receptors and had a protective effect on neuronal and synaptic function under stress. ${ }^{22}$

Creating intracellular hypoxia can induce a stress response. Acute exposure to smoke inhalation in awake rats and mice causes a hypoxic environment in the cell, an increase in reactive oxygen species, inhibition of mitochondrial respiration, and oxidative DNA damage. ${ }^{23-25}$ However, in transgenic mice with overexpression of neuroglobin (a neuroprotective globulin protein), exposure to toxic smoke inhalation caused less oxidative damage to DNA. ${ }^{23-25}$ Therefore, oxidative DNA damage caused by inhibition of mitochondrial respiratory complexes may contribute to neuronal dysfunction and progressive brain injury.

Oxidative stress may cause mitochondrial fragmentation. In two cell types (human lung adenocarcinoma cells and African green monkey kidney fibroblasts transformed with simian virus 40), laser irradiation caused oxidative stress and mitochondrial fragmentation; however, this mitochondrial damage was prevented by dehydroascorbic acid, which scavenges reactive oxygen species. ${ }^{26}$ The mitochondrial fragmentation was caused by oxidative stress and associated translocation and imbalance of profission (dynamin-related protein 1) and profusion proteins (mitofusin 2). ${ }^{26}$
Repeated daily stress causes atrophy of hippocampal CA3 pyramidal neurons in tree shrews (Tupaia belangeri). ${ }^{27}$ Male tree shrews are territorial; when subordinate male tree shrews were confined with dominant ones, the subordinates lost weight and had increased urinary cortisol levels. Apical dendrites in CA3 pyramidal cells had a decreased number of branch points and total dendritic length in subordinate tree shrews when compared with controls. ${ }^{27}$

In rats and tree shrews, repeated stress caused volume loss in the hippocampus and retraction of apical dendrites in CA3 pyramidal cells. ${ }^{28}$ Tianeptine prevented changes in hippocampal volume and preserved cell proliferation, but stress reduced neurogenesis of hippocampus neurons. ${ }^{28}$ These studies are important because one granule neuron from the entorhinal cortex of the hippocampus may innervate an average of 12 CA3 neurons, and each CA3 neuron innervates an average of 50 other CA3 neurons and 25 inhibitory cells. ${ }^{28}$ Therefore, there may be a 600 -fold amplification of excitation and 300-fold amplification of inhibition. ${ }^{28}$

The neuroprotective effect of topiramate was studied in young adult male Wistar rats in status epilepticus triggered by pilocarpine to resemble human temporal lobe epilepsy. ${ }^{29}$ In this study, the effect of large quantities of calcium on mitochondrial metabolism was evaluated from sections of the hippocampus (cornu ammonis [CA1 and CA3] and dentate gyrus) and parahippocampus. Most of the calcium accumulated in the mitochondria, as shown by a transient increase in green fluorescence of calcium. ${ }^{29}$ When larger amounts of calcium were added, the mitochondrial membrane permeability transition pore opened, causing swelling and release of cytochrome $\mathrm{c}$ from the mitochondrial intermembrane space. ${ }^{29}$ Stress models of the brain have shown that elevated cortisol levels cause histologic changes, especially in the dentate gyrus. Toxicity from excess excitation may be mediated in part by entry of calcium into the cell; the high intracellular calcium level may open the membrane permeability transition pore, depolarize the mitochondrial membrane, release cytochrome c, and cause cell death.

In rats with seizures induced with pilocarpine, intense neuronal activity is associated with loss of oxidative phosphorylation complexes I and IV located in the CA3 and CA1 pyramidal areas. ${ }^{30}$ Imaging with rhodamine showed a decreased mitochondrial membrane potential in both pyramidal subfields, but mitochondrial oxidative phosphorylation was unaltered in the dentate gyrus and parahippocampus. ${ }^{30}$ This research showed that specific tissues may be vulnerable to neurotoxicity.

Superoxide dismutase protects intracellular metabolism from oxidative stress, and there are three forms of superoxide 
dismutase in humans. The superoxide dismutase, glutathione peroxidase, reduces lipid hydroperoxides and scavenges peroxides. In a study of oxidative stress in prion-infected or uninfected hypothalamic neuronal GT1-7 cells, the infected cells showed increased lipid peroxidation and apoptosis associated with decreased glutathione-dependent and superoxide dismutase activity. ${ }^{31}$ Although this study focused on transmissible spongiform encephalopathy, it provided a stress model for hypothalamic cells and suggested that oxidative stress may dysregulate the hypothalamus. In humans, the neurohypophysis is a neural extension of the hypothalamus. The adenohypophysis secretes many proteins and is controlled by hypothalamic secretions and hypothalamic hypophyseal portal vessels.

The potent selective D1-dopamine receptor agonist, A68930, may protect the hypothalamic-pituitary-adrenal axis against unpredictable acute and chronic stress. ${ }^{32}$ Changes in fiber density and numbers of immunoreactive neurons with tyrosine hydroxylase and glucocorticoid receptors were measured in regions of the rat brain rich in dopamine and glucocorticoid receptors. Chronic unpredictable stress caused a significant decrease in the number of immunoreactive neurons located in the striatum, medial forebrain bundle, ventral tegmental area, and substantia nigra, and reduced the activity of superoxide dismutase and catalase in the cortex, striatum, and hippocampus; the unstressed control rats showed no changes. ${ }^{32}$ Therefore, the D1-dopamine agonist A68930 may have neuroprotective effects in both acute and chronic unpredictable stress. ${ }^{32}$

Mitochondrial dysfunction may also be important in mood disorder because of genetic variation or mutations in nuclear and mtDNA. Mitochondrial diseases, such as Leber's disease (hereditary optic neuropathy), have been correlated with psychiatric illness, including the effects of psychotropic drugs on mitochondria, genetic variations or mutations, polymorphisms of nuclear encoded mtDNA, and impairment of the electron transport system. ${ }^{33}$

In homoplasmy, the cell has identical copies of mtDNA; in heteroplasmy, there is more than one type of mtDNA present in the cell. ${ }^{34}$ Many patients with mood disorder have pathologic mtDNA defects and two different sets of mtDNA. The extent of heteroplasmy varies between cells and tissues. When some nuclear genes are disrupted, autosomal dominant or recessive mitochondrial disease may occur. ${ }^{34}$ Some mitochondrial diseases may cause secondary mtDNA abnormalities such as deletions or depletion of copy numbers. ${ }^{34}$ There may be a relationship between mood disorder and inflammatory processes manifested by increased plasma levels of proinflammatory cytokines and acute phase reactants, oxidative damage to red blood cell membranes, and decreased serum zinc levels. ${ }^{34}$

\section{Discussion}

The studies reviewed provide support for the hypothesis that intracellular mitochondrial metabolic dysfunction in specific brain regions is associated with MDD, either as a cause or effect. Mitochondrial dysfunction caused by oxidative stress alters intracellular metabolism and may damage mtDNA. The level of resilience or "physiologic reserve" of mitochondria to stress may explain the variation in clinical presentation and disease severity. Mitochondria replicate and respond to the energy needs of specific intracellular microenvironments. GLUT glucose transporter proteins require healthy mitochondria, and failure in one region of the cell may affect the metabolism of the entire cell. Impairment of a specific tissue may provide an explanation for the varied signs and symptoms of depression and effects on other parts of the body.

The hippocampus has interdependent neuronal connections. Histologic study of the rat hippocampus demonstrated enfolding of the hippocampus and cellular connections. ${ }^{35}$ There are four sections of the cornu ammonis. Layer 2 of the entorhinal cortex is connected through the perforant pathway to the dentate gyrus and CA3; the granular cells of the dentate gyrus are connected with CA3; the pyramidal cells of CA3 are connected with CA1; the pyramidal cells of CA1 are connected with the subiculum and deep entorhinal cortex; and the subiculum neurons are connected with the entorhinal cortex. Previous studies showed that CA3 pyramidal neurons may be compromised by the effect of physiologic stress. ${ }^{27,28}$ Stress requires mitochondrial physiologic reserve, and a change in CA3 pyramidal neurons may affect the interdependent connections of the hippocampus, especially considering the amplification effects of multiple neural connections. ${ }^{28}$

In the 19th century, there was controversy about the relationship between dementia, depression, paralysis, and brain localization, and there were differences in medical opinion about histopathologic post mortem findings, ${ }^{36,37}$ as reflected by Mairet who observed postmortem changes in the temporal lobe of melancholic patients and hypothesized that:

“...... this area might be related to primary feelings of sadness and that the nihilistic delusions were in fact secondary developments made possible by the spread of the lesion to the cortex." ${ }^{36}$ 
This 19th century debate about the presentation of melancholic patients highlighted the diverse presentation of this illness, which may be influenced by culture. Therefore, it was reasonable to infer that depression may be influenced by genetic vulnerability to stress similar to that observed in patients with sepsis. A variety of tissues may have changes in morphology and function associated with depression that may affect the clinical presentation. Chronic memory problems in depressed patients in remission may be caused by a lack of resilience of CA3 pyramidal cells.

Hippocampal function is important for verbal memory. The amygdala, which includes several nuclei, may enable long-term memory but does not store memory. The basolateral complex of the amygdala may affect long-term memory through its numerous projections. ${ }^{14}$ Memory is promoted by stress or excitement, and impaired memory during remission from mood disorder suggests that cellular metabolic damage may persist even though stability of mood has been achieved. ${ }^{18,38}$ Areas of the brain may vary in vulnerability to chronic metabolic changes in depression.

The hypothalamus and its extended neurohypophysis may affect neuroendocrine regulation. Symptoms of abnormal regulation of the hypothalamus observed with depression may include altered sleep, menstrual irregularities, altered appetite and thirst, autonomic nervous system dysfunction, and irritability. The cornu ammonis of the hippocampus connects through the fornix to the mammillary body and has efferent connections to the temporal lobe, and impairment of the hippocampus may include impaired memory, especially verbal memory.

Abnormal regulation of the prefrontal cortex may cause bradyphrenia, and amygdala dysfunction may cause abnormal emotional control. The association between MDD, decreased glial cell density, and decreased neuronal size in the supracallosal anterior cingulate cortex suggests that patients with MDD may have impaired emotional processing and impaired emotional interface with pain..$^{20,39-41}$ In addition, patients with motor cerebellar signs may also have an association between the vermis of the cerebellum, affect, and abnormal cognitive function. ${ }^{17}$

\section{Conclusion}

Although there is controversy about the biologic basis of depression, morphologic and functional abnormalities associated with mitochondrial dysfunction and oxidative stress may be associated with depression. Genetic variation or mutations in nuclear DNA and mtDNA may not be expressed and may not necessarily predict psychiatric illness.
Mitochondria are the central sources of cellular energy. Stability of cellular function requires the integrity of numerous complex enzymatic reactions. Dysfunction of mitochondrial metabolism may be a cause or effect of mood disorder. The pathologic morphology at various sites may be associated with impaired function and may enable prediction of diverse presentations of depression and chronic cognitive problems. Further understanding of mitochondrial dysfunction may help conceptualize depression as a selective tissue disorder.

\section{Disclosure}

EHT has no financial support in preparing this paper, no vested financial interests, and no connection to any research facility, pharmaceutical company, or government agency.

\section{References}

1. American Psychiatric Association. Diagnostic and Statistical Manual of Mental Disorders, 4th ed, Text Revision. Washington, DC: American Psychiatric Association; 2000.

2. Hamilton M. A rating scale for depression. J Neurol Neurosurg Psychiatry. 1960;23:56-62.

3. Fournier JC, DeRubeis RJ, Hollon SD, et al. Antidepressant drug effects and depression severity: a patient-level meta-analysis. JAMA. 2010;303(1):47-53.

4. Schildkraut JJ. The catecholamine hypothesis of affective disorders: a review of supporting evidence. Am J Psychiatry. 1965;122(5): 509-522.

5. Onions CT, editor. The Oxford Dictionary of English Etymology. Oxford, UK: Oxford University Press; 1966.

6. Brookes PS, Levonen AL, Shiva S, Sarti P, Darley-Usmar VM. Mitochondria: regulators of signal transduction by reactive oxygen and nitrogen species. Free Radic Biol Med. 2002;33(6):755-764.

7. Brookes PS, Yoon Y, Robotham JL, Anders MW, Sheu SS. Calcium, ATP, and ROS: a mitochondrial love-hate triangle. Am J Physiol Cell Physiol. 2004;287(4):C817-C833.

8. Elmore S. Apoptosis: a review of programmed cell death. Toxicol Pathol. 2007;35(4):495-516.

9. Spät A, Szanda G, Csordás G, Hajnóczky G. High- and low-calciumdependent mechanisms of mitochondrial calcium signaling. Cell Calcium. 2008;44(1):51-63.

10. Baudouin SV, Saunders D, Tiangyou W, et al. Mitochondrial DNA and survival after sepsis: a prospective study. Lancet. 2005; 366(9503):2118-2121.

11. Gould GW, Holman GD. The glucose transporter family: structure, function and tissue-specific expression. Biochem J. 1993;295(Pt 2): 329-341.

12. Rigucci S, Serafini G, Pompili M, Kotzalidis GD, Tatarelli R. Anatomical and functional correlates in major depressive disorder: the contribution of neuroimaging studies. World J Biol Psychiatry. 2010;11(2 Pt 2): 165-180.

13. Drevets WC. Functional neuroimaging studies of depression: the anatomy of melancholia. Annu Rev Med. 1998;49:341-361.

14. Bremner JD, Vythilingam M, Vermetten E, et al. Reduced volume of orbitofrontal cortex in major depression. Biol Psychiatry. 2002;51(4): 273-279.

15. Schmahmann JD. Disorders of the cerebellum: ataxia, dysmetria of thought, and the cerebellar cognitive affective syndrome. J Neuropsychiatry Clin Neurosci. 2004;16(3):367-378.

16. DelBello MP, Strakowski SM, Zimmerman ME, Hawkins JM, Sax KW. MRI analysis of the cerebellum in bipolar disorder: a pilot study. Neuropsychopharmacology. 1999;21(1):63-68. 
17. Tobe EH. Behavioral effects of incomplete temporal lobe necrosis and cerebellar damage. Biol Psychiatry. 2012;71(9):e41-e42.

18. Sheline YI, Sanghavi M, Mintun MA, Gado MH. Depression duration but not age predicts hippocampal volume loss in medically healthy women with recurrent major depression. J Neurosci. 1999;19(12): 5034-5043.

19. Rajkowska G, Miguel-Hidalgo JJ, Wei J, et al. Morphometric evidence for neuronal and glial prefrontal cell pathology in major depression. Biol Psychiatry. 1999;45(9):1085-1098.

20. Cotter D, Mackay D, Landau S, Kerwin R, Everall I. Reduced glial cell density and neuronal size in the anterior cingulate cortex in major depressive disorder. Arch Gen Psychiatry. 2001;58(6):545-553.

21. Gabay S, Achee FM, Mentes G. Some parameters affecting the activity of monoamine oxidase in purified bovine brain mitochondria. J Neurochem. 1976;27(2):415-424.

22. Gao X, Xu X, Pang J, et al. NMDA receptor activation induces mitochondrial dysfunction, oxidative stress and apoptosis in cultured neonatal rat cardiomyocytes. Physiol Res. 2007;56(5):559-569.

23. Lee HM, Reed J, Greeley GH Jr, Englander EW. Impaired mitochondrial respiration and protein nitration in the rat hippocampus after acute inhalation of combustion smoke. Toxicol Appl Pharmacol. 2009;235(2):208-215.

24. Lee HM, Greeley GH, Herndon DN, Sinha M, Luxon BA, Englander EW. A rat model of smoke inhalation injury: influence of combustion smoke on gene expression in the brain. Toxicol Appl Pharmacol. 2005;208(3): 255-265.

25. Lee HM, Greeley GH Jr, Englander EW. Transgenic overexpression of neuroglobin attenuates formation of smoke-inhalation-induced oxidative DNA damage, in vivo, in the mouse brain. Free Radic Biol Med. 2011;51(12):2281-2287.

26. Wu S, Zhou F, Zhang Z, Xing D. Mitochondrial oxidative stress causes mitochondrial fragmentation via differential modulation of mitochondrial fission-fusion proteins. FEBS J. 2011;278(6):941-954.

27. Magariños AM, McEwen BS, Flügge G, Fuchs E. Chronic psychosocial stress causes apical dendritic atrophy of hippocampal CA3 pyramidal neurons in subordinate tree shrews. J Neurosci. 1996;16(10):3534-3540

28. McEwen BS. Glucocorticoids, depression, and mood disorders: structural remodeling in the brain. Metabolism. 2005;54(5 Suppl 1): $20-23$.
29. Kudin AP, Debska-Vielhaber G, Vielhaber S, Elger CE, Kunz WS. The mechanism of neuroprotection by topiramate in an animal model of epilepsy. Epilepsia. 2004;45(12):1478-1487.

30. Kudin AP, Kudina TA, Seyfried J, et al. Seizure-dependent modulation of mitochondrial oxidative phosphorylation in rat hippocampus. Eur $J$ Neurosci. 2002;15(7):1105-1114.

31. Milhavet O, McMahon HE, Rachidi W, et al. Prion infection impairs the cellular response to oxidative stress. Proc Natl Acad Sci U S A. 2000;97(25):13937-13942.

32. Rasheed N, Ahmad A, Al-Sheeha M, Alghasham A, Palit G. Neuroprotective and anti-stress effect of A68930 in acute and chronic unpredictable stress model in rats. Neurosci Lett. 2011;504(2): 151-155.

33. Jou SH, Chiu NY, Liu CS. Mitochondrial dysfunction and psychiatric disorders. Chang Gung Med J. 2009;32(4):370-379.

34. Gardner A, Boles RG. Beyond the serotonin hypothesis: mitochondria, inflammation and neurodegeneration in major depression and affective spectrum disorders. Prog Neuropsychopharmacol Biol Psychiatry. 2011;35(3):730-743.

35. Ramón y Cajal, Santiago. Histologie du Systeme Nerveux de l'Homme et des Vertebretes. Volumes 1 and 2. Paris, France: A Maloine; 1911.

36. Berrios GE. "Depressive pseudodementia" or "melancholic dementia": a 19th century view. J Neurol Neurosurg Psychiatry. 1985;48(5):393-400.

37. Mairet A. BMJ. 1935;2(3908):1078.

38. Paré D. Role of the basolateral amygdala in memory consolidation. Prog Neurobiol. 2003;70(5):409-420.

39. Zimmermann K, Leidl C, Kaschka M, et al. Central projection of pain arising from delayed onset muscle soreness (DOMS) in human subjects. PLoS One. 2012;7(10):e47230.

40. Peyron R, Laurent B, García-Larrea L. Functional imaging of brain responses to pain. A review and meta-analysis (2000). Neurophysiol Clin. 2000;30(5):263-288.

41. Peyron R, García-Larrea L, Grégoire MC, et al. Parietal and cingulate processes in central pain. A combined positron emission tomography (PET) and functional magnetic resonance imaging (fMRI) study of an unusual case. Pain. 2000;84(1):77-87.
Neuropsychiatric Disease and Treatment

\section{Publish your work in this journal}

Neuropsychiatric Disease and Treatment is an international, peerreviewed journal of clinical therapeutics and pharmacology focusing on concise rapid reporting of clinical or pre-clinical studies on a range of neuropsychiatric and neurological disorders. This journa is indexed on PubMed Central, the 'PsycINFO' database and CAS

\section{Dovepress}

The manuscript management system is completely online and includes a very quick and fair peer-review system, which is all easy to use. Visit http://www.dovepress.com/testimonials.php to read real quotes from published authors. 